\title{
Determining the best treatment for renal cell carcinoma in young patients
}

\author{
Kenneth Pace, MD, MSc, FRCSC
}

See related article on page 610

K arakiewicz and colleagues ${ }^{1}$ have demonstrated that age at diagnosis appears to be an independent prognostic factor for cancerspecific survival in patients with renal cell carcinoma (RCC), confirming findings reported by other investigators. ${ }^{2-4}$ Unlike other sites such as the colon and prostate, younger patients presenting with RCC appear to have a better prognosis than older patients. Although one must interpret the results of retrospective studies with caution (e.g., perhaps younger patients received more detailed staging and followup imaging, or perhaps they were more likely to receive adjuvant therapy if needed), the data seem compelling. This survival benefit suggests that an age-tailored approach to managing RCC is important. Given the favourable survival data for younger patients, the importance of nephron-sparing surgery increases, even in cases where it may not be imperative. This is particularly true when combined with data suggesting that long-term renal function is superior following nephronsparing surgery $y^{5}$ and cancer-specific survival is equivalent to open surgery. Although partial nephrectomy remains the gold standard form of nephron-sparing surgery, the role for other renal ablative technologies such as radio-frequency ablation, cryotherapy, high-intensity focused ultrasound and the Gamma Knife remains to be defined - particularly in the treatment of the disease in younger patients. Minimizing patient morbidity while maximizing survival and long-term cure rates is important to all patients, but it is doubly important in younger patients.

In contrast, older patients who appear to have a poorer prognosis might benefit from adjuvant systemic therapies, even without evidence of metastatic disease. Once again, this hypothesis must be tested in randomized trials before widespread implementation. Older patients might also benefit from closer follow-up imaging surveillance than younger patients. The role for extended lymphadenectomy is still being debated, but age might be another useful preoperative predictor of which patients are more likely to benefit. Based on this data, younger patients with lower stage tumours might not require an extended lymphadenectomy, whereas older patients with the same disease burden might benefit. Again, this hypothesis would need to be tested in other trials before it could become routine practice.

Renal cell carcinoma remains a clinically perplexing and heterogeneous disease. Patient age (when combined with other factors such as radiographic appearance, stage and functional status) is one more variable that clinicians should incorporate into their decision-making algorithms to determine the most appropriate treatment for these patients.

Assistant professor, Division of Urology, Department of Surgery, Co-director, Fellowship in Endourology and Laparoscopy, St. Michael's Hospital, University of Toronto, Toronto, Ont.

Competing interests: None declared.

\section{References}

1. Karakiewicz Pl, Jeldres C, Suardi N, et al. Age at diagnosis is a determinant factor of renal cell carcinoma-specific survival in patients treated with nephrectomy. Can Urol Assoc J 2008;2:610-7.

2. Sanchez-Ortiz RF, Rosser CJ, Madsen LT, et al. Young age is an independent prognostic factor for survival of sporadic renal cell carcinoma. J Urol 2004;171:2160-5.

3. Taccoen X, Valeri A, Descotes IL, et al. Renal cell carcinoma in adults 40 years old or less: young age is an independent prognostic factor for cancerspecific survival. Eur Urol 2007;51:980-7.

4. Verhoest $G$, Veillard D, Guille $F$, et al. Relationship between age at diagnosis and clinicopathologic features of renal cell carcinoma. Eur Urol 2007:51:1298-304.

5. Clark AT, Breau RH, Morash C, et al. Preservation of renal function following partial or radical nephrectomy using 24-hour creatinine clearance. Eur Urol 2008;54:143-9.

Correspondence: Dr. Kenneth Pace, 61 Queen St. E. Suite 9-106, St. Michael's Hospital, University of Toronto, Toronto ON M5C 2T2; kenneth.pace@utoronto.ca 